\title{
On the spectra of some $g$-circulant matrices and applications to nonnegative inverse eigenvalue problem
}

\author{
Enide Andrade \\ CIDMA-Center for Research and Development in Mathematics and Applications \\ Department of Mathematics, University of Aveiro, 3810-193, Aveiro, Portugal. \\ Luis Arrieta \\ Departamento de Matemáticas, Universidad Católica del Norte, Av. Angamos 0610 \\ Antofagasta, Chile. \\ Cristina Manzaneda \\ Departamento de Matemáticas, Facultad de Ciencias. Universidad Católica del Norte. \\ Av. Angamos 0610 Antofagasta, Chile. \\ María Robbiano* \\ Departamento de Matemáticas, Universidad Católica del Norte, Av. Angamos 0610 \\ Antofagasta, Chile.
}

\begin{abstract}
A $g$-circulant matrix $A$, is defined as a matrix of order $n$ where the elements of each row of $A$ are identical to those of the previous row, but are moved $g$ positions to the right and wrapped around. Using number theory, certain spectra of $g$-circulant real matrices are given explicitly. The obtained results are applied to Nonnegative Inverse Eigenvalue Problem to construct nonnegative, $g$-circulant matrices with given appropriated spectrum. Additionally, some $g$-circulant marices are reconstructed from its main diagonal entries.
\end{abstract}

Keywords: Nonnegative inverse eigenvalue problem, nonnegative matrix, circulant matrix, $g$-circulant matrix, permutative matrix

\footnotetext{
*Corresponding author

Email addresses: enide@ua.pt (Enide Andrade), luis.arrieta01@ucn.cl (Luis Arrieta), cmanzaneda@ucn.cl (Cristina Manzaneda), mrobbiano@ucn.cl (María
} Robbiano ) 


\section{Introduction}

A permutative matrix is a square matrix where each row is a permutation of its first row. A circulant matrix is a matrix where each row its a right cyclic shift in one place of the previous one. A $g$-circulant matrix is a matrix where each row its a right cyclic shift in $g$-places of the previous row. Circulant matrices and $g$-circulant matrices are permutative. An $n$-tuple of complex numbers,

$$
\Sigma=\left(\lambda_{1}, \lambda_{2}, \ldots, \lambda_{n}\right)
$$

is said to be realizable if there exists a nonnegative matrix $A$ of order $n$ such that its components are the eigenvalues of $A$. The study of circulant matrices and their use in realizing lists of complex numbers has produced many results. In this work we propose to carry out similar research for $g$ circulant matrices. The Nonnegative Inverse Eigenvalue Problem (NIEP) is a problem to determine necessary and sufficient conditions for a list of $n$ complex numbers to be realizable. If the list $\Sigma$ is realizable by a nonnegative matrix $A$, then we say that $A$ realizes $\Sigma$ or it is a realizing matrix for $\Sigma$. Some results can be seen in $[10,11,12]$. This very difficult problem attracted the attention of many authors over the last 50 years, and it was firstly considered by Suleĭmanova in 1949 (see [28]). Some partial results were obtained but it is still an open problem for $n \geq 5$.

In [15] the problem was solved for $n=3$ and for matrices with trace zero of order $n=4$ and $n=5$, the problem have been solved by [23] and Laffey and Meehan in [20], respectively.

In its general form it was studied in $[3,7,8,13,14,15,24,25,27]$. When the nonnegative realizing matrix $A$ is required to be symmetric the problem is designated Symmetric Nonnegative Inverse Eigenvalue Problem (SNIEP) and it is also an open problem. It also has been a problem that had called much attention, see for instance, $[5,8,16,27]$. Another variant of this problem is to find lists of $n$ real numbers that can be lists of eigenvalues of nonnegative matrices of order $n$ and it is called the Real Nonnegative Inverse Eigenvalue Problem (denoted by RNIEP). Some results can be seen, for instance, in $[8,15]$. Similarly, in the structured NIEP the matrix that realizes the list is structured. For instance, the matrix can be symmetric, Toeplitz, Hankel, 
circulant, $g$-circulant, normal, permutative, etc., see $[1,5,8,16,18,22]$ and the references cited therein.

In this paper we deal with structured matrices, in particular, circulant and $g$-circulant matrices. The study of a list of complex numbers satisfying sufficient conditions in order that it can be the spectrum of a nonnegative permutative matrix allows us to answer the question if there exist sufficient conditions so that this the list of complex numbers can be realizable by a type of permutative matrix and at same time realizable by another type of permutative matrix. The paper is organized as follows: in Section 2 the definition of $g$-circulant matrix is recalled and some of its properties are presented. In Section 3 it is determined, in an explicit way, the spectrum of a subclass of real $g$-circulant matrices, namely circulant matrices of order $n$ where $n$ is a prime integer such that $(n, g)=1$, where throughout the text, $(a, b)$ denotes the greatest common divisor of integers $a$ and $b$.

Moreover, sufficient conditions in order that a given list can be taken as the spectrum of a nonnegative $g$-circulant matrix are given. Additionally, a necessary and sufficient condition for certain lists to become realizable by a $g$-circulant matrix is presented.

In Section 4 it is shown that for certain positive integers $n$ and $g$, a $g$ circulant matrix of order $n$ is completely determined by its diagonal entries. The following notation will be used. A square nonnegative matrix $A$ is denoted by $A \geq 0 . \Sigma(A)$ denotes the set of eigenvalues of a square matrix $A$. The transpose of $A$ is denoted by $A^{T}$. The trace of $A$ is $\operatorname{tr}(\mathrm{A})$. The set of the invertible elements in the set of the residue classes congruence $(\bmod n), \mathbb{Z}_{n}$, will be denoted by $U_{n}$.

\section{2. $g$-circulant matrices and some properties}

Circulant matrices are an important class of matrices and have many connections to physics, probability and statistics, image processing, numerical analysis, number theory and geometry. Many properties of these matrices can be found in, for instance, $[4,9]$. In terms of notation, these matrices are perfectly identified by its first row and we just write $C=C(\mathbf{c})=\operatorname{circ}\left(c_{0}, \ldots, c_{n-1}\right)$.

Remark 1. [25] If $\lambda=\left(\lambda_{0}, \lambda_{1}, \ldots, \lambda_{n-1}\right)^{T}$ is the vector of eigenvalues of a real circulant matrix $C$, then $\lambda_{0}$ is a real number and

$$
\lambda_{n-k}=\bar{\lambda}_{k}, \quad 1 \leq k \leq \frac{n}{2},
$$


where $n$ is even. We note that, in this case, $\lambda_{\frac{n}{2}}$ is a real number. Analogously if $n$ is odd then,

$$
\lambda_{n-k}=\bar{\lambda}_{k}, \quad 1 \leq k \leq \frac{n-1}{2} .
$$

In this section a generalization of circulant matrices is recalled, namely $g$ circulant matrices. Some known properties of these interesting matrices are pointed out.

Definition 2. [4] A g-circulant matrix of order $n$, or simply g-circulant, is a matrix in the following form:

$$
\begin{aligned}
A & =g-\operatorname{circ}\left(a_{0}, a_{1}, \ldots, a_{n-1}\right) \\
& =\left(\begin{array}{ccccc}
a_{0} & a_{1} & a_{2} & \ldots & a_{n-1} \\
a_{n-g} & a_{n-g+1} & a_{n-g+2} & & a_{n-g-1} \\
a_{n-2 g} & a_{n-2 g+1} & a_{n-2 g+2} & & a_{n-2 g-1} \\
\vdots & & \ldots & \ddots & \vdots \\
a_{g} & a_{g+1} & & \ldots & a_{g-1}
\end{array}\right) .
\end{aligned}
$$

Here the subscripts are taken $(\bmod n)$, as for the circulant matrices.

Remark 3. [4]

- If $g=1$ a g-circulant matrix is a circulant matrix.

- If $0 \leq g \leq n$, each row of $A$ is a right cyclic shift in $g$-places (or it is a left cyclic shift in $(n-g)$-places) to the preceding row.

- If $g \geq n$ a cyclic shift in $g$-places is the same cyclic shift in $g(\bmod \mathrm{n})$ places.

- By convention if $g$ is negative a right cyclic shift in g-places is equivalent to a left cyclic shift in $(-g)$-places. In consequence, for any integers $g$ and $g^{\prime}$, if $g \equiv g^{\prime}(\bmod \mathrm{n})$ then a $g$-circulant and a $g^{\prime}$-circulant with the same first row are equal.

- In particular if $g=n-1, a(-1)$-circulant matrix is obtained, see [4]. 
Many examples of $g$-circulant matrices can be also found in [4].

The entries of a $g$-circulant matrix can also be specified. Let $A=$ $\left(a_{i j}\right)_{1 \leq i, j \leq n}$, from [4], $A$ is a $g$-circulant matrix if and only if

$$
a_{i j}=a_{i+1, j+g}, \quad 1 \leq i, j \leq n,
$$

In an equivalent way, if the matrix $A=\left(a_{i j}\right)$ is a $g-\operatorname{circ}\left(a_{0}, \ldots, a_{n-1}\right)$ then

$$
a_{i j}=a_{(j-1)-(i-1) g}, \quad 1 \leq i, j \leq n,
$$

In the right hand expression, the subscripts are taken $(\bmod n)$. Using the expression above, that is written for a $g-\operatorname{circ}\left(a_{0}, \ldots, a_{n-1}\right), A$, one can say that $A$ is symmetric if and only if $(j-1)-(i-1) g \equiv(i-1)-(j-1) g(\operatorname{modn})$ which is equivalent to $(j-i)(g+1) \equiv 0(\bmod n)$. The previous expression is also equivalent to either $(j-i) \equiv 0(\bmod n) \forall \mathrm{i}, \mathrm{j}$ or $(g+1) \equiv 0(\bmod \mathrm{n})$, as $n$ is prime. Thus, $g \equiv n-1(\bmod \mathrm{n})$, as $g$ can be chosen $0 \leq g \leq n-1$. Therefore $g=n-1$. We conclude that a $g$-circulant matrix of order $n$, with $n$ prime is symmetric if and only if $g \equiv n-1(\bmod n)$.

Let $g \geq 0$. The $g$-circulant matrices of order $n$ can be partitioned into two types, namely either $(n, g)=1$ or $(n, g)>1$. It is clear that all the rows of a $g$-circulant matrix are distinct if and only if $(n, g)=1$, see [4] and, this is the case that we study in this work. In this case, the rows of the $g$-circulant matrix can be permuted in such a way to re-obtain a classical circulant matrix. The same reasoning can be done for columns.

If $(n, g)=1$, the unique solution of the equation $g x \equiv 1(\bmod n)$ will be designated as $g^{-1}$, [6]. Note that, from [4], if $A$ is a non singular $g$-circulant matrix then $A^{-1}$ is a $g^{-1}$-circulant matrix.

Let $Q_{g}=g-\operatorname{circ}(1,0, \ldots, 0)$. Note that $Q_{g}$ is a permutation matrix if and only if $(n, g)=1$. The next result can be seen in [4].

Proposition 4. [4] The matrix $A$ is g-circulant if and only if $A$ is of the form

$$
A=Q_{g} C
$$

where $C$ is a circulant matrix whose first row coincide with the first row of A.

The eigenvalues of a $g$-circulant matrix $A$ are obtained in [26] by using the equality in (4). 


\section{Characterizing the spectra of certain $g$-circulant matrices}

In this section, it is determined in an explicit way, the spectrum of a subclass of real $g$-circulant matrices. We start presenting a result from [26] with explicit formulas for the eigenvalues of a $g$-circulant matrix. The authors, in [26], provided closed form expressions of the eigenvalues of $g$-circulant matrices that we can see below. By convenience, only in the following paragraph the subscripts are written as $(\bmod n)$ as they were considered in $[26]$. Therefore, from [26] we have:

- When $g=1$ then the $g$-circulant matrix is a circulant matrix and its eigenvalues can be seen in [4].

- When $g=0$ then all rows of the 0-circulant matrix, are equal and the matrix has $n-1$ zero eigenvalues and one eigenvalue $\lambda=\sum_{r=0}^{n-1} a_{r}$, where $\left[a_{0}, a_{1}, \ldots, a_{n-1}\right]^{T}$ is the first (and the unique) row of the 0 circulant matrix $C$.

- When $(n, g)=1$ with $g \notin\{0,1\}$, the modulus of the eigenvalues of the $g$-circulant matrix $A$ are given by

$$
\left|\beta_{j}(A)\right|=\left|\left(\prod_{k=0}^{s-1} \lambda_{\left(g^{k} j\right)(\bmod \mathrm{n})}\right)^{\frac{1}{s}}\right|, j=0, \ldots, n-1,
$$

where a positive integer $s$ is such that $g^{s} \equiv 1(\bmod n)$, and $D=$ $\operatorname{diag}\left(\lambda_{0}, \ldots, \lambda_{n-1}\right)$ is the diagonal of the eigenvalues of the circulant matrix $C=Q_{g}^{-1} A$.

- When $(n, g) \neq 1$ and $g \notin\{0,1\}$ then the $g$-circulant matrix $A$ has $n-n_{g}$ eigenvalues equal to zero and the remaining $n_{g}$ eigenvalues are the eigenvalues of a matrix $A_{g}$ that is a $\hat{g}$-circulant matrix of order $n_{g}$ where $\hat{g} \equiv g(\bmod \mathrm{n})$, whose elements are:

$$
\left(A_{g}\right)_{j, k}=\sum_{t=0}^{(n, g)-1} a_{\left(j+t n_{g}-g k\right)(\bmod \mathrm{n})}, j, k=0, \ldots, n_{g}-1 .
$$

Next, we obtain the spectrum of a class of $g$-circulant matrices namely, the spectrum of circulant matrices of order $n$ where $n$ is a prime integer such that $(n, g)=1$.

The following definition and notation from group theory are recalled (see $[6])$. 
Definition 5. [6] Let $G$ and $H$ be a group and subgroup of $G$, respectively. The subgroup $H$ is a normal subgroup of $G$ whenever for all $g \in G$, the condition $g^{-1} \mathrm{Hg}=H$ holds. If $G$ is a commutative group all subgroup of $G$ is normal.

The notation $H \unlhd G$, means that $H$ is a normal subgroup of $G$. The order, or cardinality, of a finite group $H$ is written as $|H|$. Let $\mathbb{Z}_{n}$ be the set of residues classes modulo $n$ and $U_{n}$ be the multiplicative group of its units, that is, the invertible elements in $\mathbb{Z}_{n}$. Note that, if $n$ is a prime number, then $\mathbb{Z}_{n}$ is a field and therefore, all of its units are the nonzero elements in $\mathbb{Z}_{n}$. The cyclic multiplicative subgroup $\left\{1, g, g^{2}, \ldots\right\}$ in $U_{n}$ is denoted by $\langle g\rangle$. The following result is well known (see [6]).

Theorem 6. [6] Let $n$ be a prime integer. Let $n$ and $g$ be positive integers such that $(n, g)=1$. Consider the binary relation in $U_{n}, \sim$, defined by $h_{1} \sim h_{2}$ if and only if $h_{1}^{-1} h_{2} \in\langle g\rangle$. Then $\sim$ is an equivalence relation, where the equivalence class of $h \in U_{n}$ is given by

$$
h\langle g\rangle=\left\{h, h g, h g^{2}, \ldots\right\} .
$$

As all these classes are disjoint, define a partition of $U_{n}$ and have the same cardinality, then,

$$
U_{n}=\bigcup_{\ell=0}^{k-1} h_{\ell}\langle g\rangle,
$$

where, $h_{0}=1, h_{j}, h_{\ell} \notin\langle g\rangle$, for $j \neq \ell$, and $k$ is an integer such that

$$
\frac{n-1}{k}=|\langle g\rangle| .
$$

In order to search for the additive inverses we need study two cases: $\langle g\rangle \unlhd U_{n}$ is even and odd, respectively.

3.1. The order of the subgroup $\langle g\rangle \unlhd U_{n}$ is even

The following result gives us the additive inverses in $\langle g\rangle$ whenever $|\langle g\rangle|=$ $d=2 m$. The case $g$ odd will be studied at Subsection 3.2.

Theorem 7. Let $n>2$ be a prime integer. Let $n$ and $g$ be positive integers such that $(n, g)=1$, if $|\langle g\rangle|=d=2 m$, then

$$
g^{m} \equiv-1(\bmod \mathrm{n}) .
$$


Proof. Note that, $g^{d}=g^{2 m} \equiv 1(\bmod n)$ holds. If the condition in $(7)$ does not hold, by the identity

$$
g^{m}\left(1+g^{m}\right) \equiv\left(1+g^{m}\right)(\bmod \mathrm{n})
$$

we obtain that

$$
g^{m} \equiv 1(\bmod \mathrm{n})
$$

which is a contradiction, as $|\langle g\rangle|=2 m$.

The following result gives the list of the eigenvalues of a $g$-circulant matrix $A$ when the order of the subgroup $\langle g\rangle$ is even.

Theorem 8. Let $n>2$ be a prime integer. Let $n$ and $g$ be positive integers such that $(n, g)=1$, and $|\langle g\rangle|=d=2 m$, with $d \geq 2$. Moreover, suppose that the equalities in (5) and in (6) hold and let

$$
\varphi=\exp \left(\frac{2 \pi \mathrm{i}}{\mathrm{d}}\right)
$$

then the g-circulant matrix $A$ of order $n=k d+1$ has as eigenvalues the elements in following list,

$$
\begin{array}{cccccc}
\beta_{0} & \beta_{1} & \beta_{1} \varphi & \beta_{1} \varphi^{2} & \ldots & \beta_{1} \varphi^{d-1} \\
& \beta_{2} & \beta_{2} \varphi & \beta_{2} \varphi^{2} & \ldots & \beta_{2} \varphi^{d-1} \\
\vdots & & & & \\
& \beta_{k} & \beta_{k} \varphi & \beta_{k} \varphi^{2} & \ldots & \beta_{k} \varphi^{d-1}
\end{array}
$$

with for $t=0,1, \ldots, k-1$,

$$
\beta_{t+1}=\left(\lambda_{h_{t}} \lambda_{h_{t} g} \lambda_{h_{t} g^{2}} \cdots \lambda_{h_{t} g^{d-1}}\right)^{\frac{1}{d}} \in \mathbb{R}
$$

are nonnegative real numbers where

$$
\lambda_{0}, \lambda_{1}, \ldots, \lambda_{n-1},
$$

are the eigenvalues of the circulant matrix

$$
C=Q_{g^{-1}} A
$$

Proof. Note that by Theorem $7,-1 \equiv g^{m}(\bmod n) \in\langle g\rangle$, then if $g^{w} \in$ $\langle g\rangle$, for some $w$, then $-g^{w} \equiv g^{(m+w)}(\bmod n)$ implying that $-g^{w} \in\langle g\rangle$. 
Similarly, if $h g^{w} \in h\langle g\rangle$ then $-h g^{w} \equiv h g^{(m+w)}$ (mod n) implying that $-h g^{w} \in h\langle g\rangle$. We claim that,for $t=0,1, \ldots, k-1$, if $\lambda_{h_{t} g^{w}} \in \mathcal{I}_{t}=$ $\left\{\lambda_{h_{t}}, \lambda_{h_{t} g}, \lambda_{h_{t} g^{2}}, \cdots, \lambda_{h_{t} g^{d-1}}\right\}$ then $\bar{\lambda}_{h_{t} g^{w}}=\lambda_{-h_{t} g^{w}} \in \mathcal{I}_{t}$ since $-h_{t} g^{w} \in h_{t}\langle g\rangle$, as previously seen. Thus, as $d$ is even, from the previous observations, one can see that the factors in the product under the root of the definition of $\beta_{t+1}$ in (10) have the form $\left|\lambda_{h_{t} g^{w}}\right|^{2}$ implying that $\beta_{t+1} \geq 0$. On the other hand, by the proof in [26, Lemma 4.1] the eigenvalues of $A$ are the $d$-th roots of the product $\lambda_{h_{t}} \lambda_{h_{t} g} \lambda_{h_{t} g^{2}} \cdots \lambda_{h_{t} g^{d-1}}$ which, for each $t=0,1, \ldots, k-1$ are given by the list in (9). Thus the statement holds.

Remark 9. Since $d$ is even and (10) holds, the numbers $\beta_{1}, \beta_{2}, \ldots, \beta_{k}$ are nonnegative real numbers. However, the condition $2 m=d \geq 2$ implies that $m \leq d-1$, and therefore, for $t=0,1, \ldots, k-1$, the numbers

$$
-\beta_{t+1}=\beta_{t+1} \exp \left(\frac{2 \mathrm{~m} \pi \mathrm{i}}{2 \mathrm{~m}}\right)=\beta_{\mathrm{t}+1} \varphi^{\mathrm{m}}
$$

also belongs to the list in (9).

Using the previous theorem and the list of elements displayed below, jointly with Remark 9, it is possible to solve the following inverse eigenvalue problem.

Problem 10. Given the real numbers $\beta_{0}, \beta_{1}, \beta_{2}, \ldots, \beta_{k}$ find a real square matrix $A$ whose spectrum contains the real numbers $\beta_{0}, \pm \beta_{1}, \pm \beta_{2}, \ldots, \pm \beta_{k}$.

Let

$$
\tau=\exp \left(\frac{2 \pi i}{n}\right)
$$

In fact, in order to solve Problem10 we need to consider the following auxiliary list depicted in the table below:

\begin{tabular}{|c|c|c|c|l|c|}
\hline$\lambda_{0}$ & $\lambda_{1}$ & $\lambda_{g}$ & $\lambda_{g^{2}}$ & $\cdots$ & $\lambda_{g^{d-1}}$ \\
\hline & $\lambda_{h_{1}}$ & $\lambda_{h_{1} g}$ & $\lambda_{h_{1} g^{2}}$ & $\cdots$ & $\lambda_{h_{1} g^{d-1}}$ \\
\hline & $\vdots$ & & & & \\
\hline & $\lambda_{h_{k-1}}$ & $\lambda_{h_{k-1} g}$ & $\lambda_{h_{k-1} g^{2}}$ & $\cdots$ & $\lambda_{h_{k-1} g^{d-1}}$ \\
\hline
\end{tabular}

\begin{tabular}{|c|c|c|c|c|c|}
\hline$\beta_{0}$ & $\beta_{1} \tau$ & $\beta_{1} \tau^{g}$ & $\beta_{1} \tau^{g^{2}}$ & $\cdots$ & $\beta_{1} \tau^{g^{d-1}}$ \\
\hline$\vdots$ & $\beta_{2} \tau^{h_{1}}$ & $\beta_{2} \tau^{h_{1} g}$ & $\beta_{2} \tau^{h_{1} g^{2}}$ & $\cdots$ & $\beta_{2} \tau^{h_{1} g^{d-1}}$ \\
\hline & $\vdots$ & $\vdots$ & $\vdots$ & $\vdots$ & $\vdots$ \\
\hline & $\beta_{k} \tau^{h_{k-1}}$ & $\beta_{k} \tau^{h_{k-1} g}$ & $\beta_{k} \tau^{h_{k-1} g^{2}}$ & $\cdots$ & $\beta_{k} \tau^{h_{k-1} g^{d-1}}$ \\
\hline
\end{tabular}


Remark 11. It is well known (see[6]) that the condition $|\langle g\rangle|=d$ implies that $d$ is the smallest positive integer such that $g^{d} \equiv 1(\bmod \mathrm{n})$ and by the identity $(1-g)\left(1+g+g^{2}+\cdots+g^{d-1}\right) \equiv\left(1-g^{d}\right)(\bmod \mathrm{n})$

we can check that

$$
1+g+g^{2}+\cdots+g^{d-1} \equiv 0(\bmod \mathrm{n})
$$

as $n$ is prime.

For the spectrum in (13) one can see that for $t=1,2, \ldots, k-1$

$$
\begin{aligned}
\left(\lambda_{h_{t}} \lambda_{h_{t} g} \lambda_{h_{t} g^{2}} \cdots \lambda_{h_{t} g^{d-1}}\right)^{\frac{1}{d}} & =\left(\beta_{t+1}^{d} \tau^{h_{t}\left(1+g+g^{2}+\cdots+g^{d-1}\right)}\right)^{\frac{1}{d}} \\
& =\beta_{t+1} \tau^{h_{t}(0)}=\beta_{t+1} .
\end{aligned}
$$

Moreover, it is clear that

$$
\begin{aligned}
\bar{\lambda}_{h_{t} g^{w}} & =\overline{\beta_{t+1} \tau^{h_{t} g^{w}}} \\
& =\beta_{t+1} \tau^{n-h_{t} g^{w}} \\
& =\beta_{t+1} \tau^{-h_{t} g^{w}} \\
& =\beta_{t+1} \tau^{h_{t} g^{m+w}} \\
& =\lambda_{h_{t} g^{m+w}}
\end{aligned}
$$

with

$$
m=\frac{d}{2}
$$

The next example illustrates Theorem 8.

Example 12. Let us suppose that $(n, g)=(13,5)$, denote $\varphi=\exp \left(\frac{2 \pi \mathrm{i}}{4}\right)=\mathrm{i}$. Therefore,

$$
\begin{aligned}
\langle g\rangle & =\langle 5\rangle=\{1,5,8,12\} \\
2\langle g\rangle & =2\langle 5\rangle=\{2,3,10,11\} \\
4\langle g\rangle & =4\langle 5\rangle=\{4,7,6,9\} .
\end{aligned}
$$

Then, the spectrum of a 5-circulant matrix $A=Q_{5} C$ of order 13 where $C$ is a circulant matrix with eigenvalues $\lambda_{0}, \lambda_{1}, \lambda_{2}, \lambda_{3}, \lambda_{4}, \lambda_{5}, \lambda_{6}, \lambda_{7}, \lambda_{8}, \lambda_{9}, \lambda_{10}, \lambda_{11}, \lambda_{12}$ is

$$
\beta_{0}:=\lambda_{0}
$$




$$
\begin{aligned}
& \beta_{1}:=\left(\lambda_{1} \lambda_{5} \lambda_{8} \lambda_{12}\right)^{\frac{1}{4}} \\
& \beta_{2}:=\left(\lambda_{2} \lambda_{3} \lambda_{10} \lambda_{11}\right)^{\frac{1}{4}} \\
& \beta_{3}:=\left(\lambda_{4} \lambda_{7} \lambda_{6} \lambda_{9}\right)^{\frac{1}{4}}
\end{aligned}
$$

together with the elements displayed in the following table:

\begin{tabular}{|c|c|c|}
\hline$\beta_{1} \varphi$ & $\beta_{1} \varphi^{2}$ & $\beta_{1} \varphi^{3}$ \\
\hline$\beta_{2} \varphi$ & $\beta_{2} \varphi^{2}$ & $\beta_{2} \varphi^{3}$ \\
\hline$\beta_{3} \varphi$ & $\beta_{3} \varphi^{2}$ & $\beta_{3} \varphi^{3}$ \\
\hline
\end{tabular}$=$\begin{tabular}{|l|l|l|}
\hline$i \beta_{1}$ & $-\beta_{1}$ & $-i \beta_{1}$ \\
\hline$i \beta_{2}$ & $-\beta_{2}$ & $-i \beta_{2}$ \\
\hline$i \beta_{3}$ & $-\beta_{3}$ & $-i \beta_{3}$ \\
\hline
\end{tabular}

The following result gives a sufficient condition on the elements $\beta_{0}, \beta_{1}, \beta_{2}, \ldots, \beta_{k}$ $\in \mathbb{R}$ in order to construct a $g$-circulant nonnegative matrix $A$ with those elements as a sublist of the list of its eigenvalues.

Theorem 13. Let $n$ and $g$ be positive integers with identical conditions as in the statement of Theorem 8. Consider the list of elements as in (9). Note that, with the reference (9) $\varphi$ is also given.

$$
\Sigma=\left(\beta_{0}, \beta_{1}, \beta_{1} \varphi, \ldots \beta_{1} \varphi^{d-1}, \beta_{2}, \beta_{2} \varphi, \ldots \beta_{2} \varphi^{d-1}, \ldots, \beta_{k}, \beta_{k} \varphi, \ldots \beta_{k} \varphi^{d-1}\right),
$$

with $\beta_{0}, \beta_{1}, \beta_{2}, \ldots, \beta_{k}$, arbitrary nonnegative real numbers. Then a sufficient condition to find a g-circulant real nonnegative matrix $A$ whose spectrum is formed by the components $\Sigma$ is

$$
\frac{\beta_{0}}{2} \geq-\left(\beta_{1} \sum_{\ell=0}^{m-1} \cos \frac{2(1-j) g^{\ell} \pi}{n}+\beta_{2} \sum_{\ell=0}^{m-1} \cos \frac{2 h_{1}(1-j) g^{\ell} \pi}{n}+\cdots+\beta_{k} \sum_{\ell=0}^{m-1} \cos \frac{2 h_{k-1}(1-j) g^{\ell} \pi}{n}\right) .
$$

\section{Proof.}

Let us consider the auxiliary list of $\lambda^{\prime}$ s defined by (13). Then by Theorem $\left[9\right.$, Theorem 3.1], the first row $\left(c_{0}, c_{1}, \ldots, c_{n-1}\right)$ of the circulant matrix of order $n$ whose spectrum is formed by the elements $\lambda^{\prime} \mathrm{s}$ in the list (13) is identified as:

$$
c_{j}=\frac{1}{n} \sum_{\ell=0}^{n-1} \lambda_{\ell} \tau^{-\ell j}, \quad 0 \leq j \leq n-1 .
$$

Replacing the $\lambda^{\prime}$ s by the given spectrum and following the equalities in 
(13) we have

$$
\begin{aligned}
c_{j} & =\frac{1}{n}\left(\beta_{0}+\beta_{1} \sum_{\ell=0}^{d-1} \tau^{g^{\ell}} \tau^{-g^{\ell} j}+\beta_{2} \sum_{\ell=0}^{d-1} \tau^{h_{1} g^{\ell}} \tau^{-h_{1} g^{\ell} j}+\cdots+\beta_{k} \sum_{\ell=0}^{d-1} \tau^{h_{k-1} g^{\ell}} \tau^{-h_{k-1} g^{\ell} j}\right) \\
& =\frac{1}{n}\left(\beta_{0}+\beta_{1} \sum_{\ell=0}^{m-1}\left(\tau^{g^{\ell}(1-j)}+\tau^{-g^{\ell}(1-j)}\right)+\beta_{2} \sum_{\ell=0}^{m-1}\left(\tau^{h_{1} g^{\ell}(1-j)}+\tau^{-h_{1} g^{\ell}(1-j)}\right)+\cdots\right. \\
& \left.+\beta_{k} \sum_{\ell=0}^{m-1}\left(\tau^{h_{k-1} g^{\ell}(1-j)}+\tau^{-h_{k-1} g^{\ell}(1-j)}\right)\right) \\
& =\frac{1}{n}\left(\beta_{0}+2 \beta_{1} \sum_{\ell=0}^{m-1} \cos \frac{2(1-j) g^{\ell} \pi}{n}+2 \beta_{2} \sum_{\ell=0}^{m-1} \cos \frac{2 h_{1}(1-j) g^{\ell} \pi}{n}+\cdots\right. \\
& \left.+2 \beta_{k} \sum_{\ell=0}^{m-1} \cos \frac{2 h_{k-1}(1-j) g^{\ell} \pi}{n}\right) .
\end{aligned}
$$

Imposing the nonnegativity of these coefficients, the inequality in (14) is obtained.

Remark 14. By the proof of Theorem 13, one can conclude that given the nonnegative list $\mathbf{b}=\left(\beta_{0}, \beta_{1}, \ldots, \beta_{k}\right)^{T}$ the coefficient vector

$$
\mathbf{c}=\left(c_{0}, c_{1}, \ldots, c_{n-1}\right)^{T}
$$

of the first row of the g-circulant matrix whose spectrum contains the elements

$$
\beta_{0}, \pm \beta_{1}, \ldots, \pm \beta_{k}
$$

are given by

$$
\mathbf{c}=\frac{1}{n} N \mathbf{b}
$$

where $N=\left(n_{u v}\right)$ is the $n \times(k+1)$ matrix whose entries are defined by

$$
n_{u v}=\left\{\begin{array}{ccc}
1 & \text { if } v=1 \text { and } 1 \leq u \leq n \\
2 \sum_{\ell=0}^{m-1} \cos \frac{2(u-1) h_{(v-2)} g^{\ell} \pi}{n} & \text { if } 2 \leq v \leq k+1 \text { and } 1 \leq u \leq n .
\end{array}\right.
$$

Corollary 15. Consider $n$ a prime integer, $g=n-1$, and the list $\mathbf{b}=$ $\left(\beta_{0}, \beta_{1}, \ldots, \beta_{\frac{n-1}{2}}\right)^{T}$. Then, a $(n-1)$-circulant matrix $A$ of order $n$ whose spectrum contains the elements in the following set

$$
\left\{\beta_{0}, \pm \beta_{1}, \ldots, \pm \beta_{\frac{n-1}{2}}\right\}
$$


is an $(n-1)$-circulant matrix whose first row $\mathbf{c}=\left(c_{0}, c_{1}, \ldots, c_{n-1}\right)^{T}$ has coefficients such that

$$
\begin{gathered}
\frac{n c_{j}}{2}-\frac{\beta_{0}}{2}-\beta_{1}\left(\cos \frac{2(1-j) \pi}{n}+\cos \frac{2(1-j)(n-1) \pi}{n}\right)= \\
\beta_{2}\left(\cos \frac{4(1-j) \pi}{n} \cos \frac{4(1-j)(n-1) \pi}{n}\right)+\cdots+ \\
\beta_{\frac{n-1}{2}}\left(\cos \frac{(n-1)(1-j) \pi}{n}+\cos \frac{(n-1)^{2}(1-j) \pi}{n}\right) .
\end{gathered}
$$

with $0 \leq j \leq n$.

Proof. Since $(n, g)=(n, n-1)=1$ and $\langle g\rangle=\langle n-1\rangle=\{1, n-1\} \unlhd U_{n}$, we have $d=2$ and then $m=1$. Moreover, there are $k=\frac{n-1}{2}$ equivalence classes, of the form $h\langle g\rangle$, namely, the classes represented by the components of the following vector $\left(h_{0}, h_{1}, \ldots, h_{\frac{n-3}{2}}\right)=\left(1,2, \ldots, \frac{n-1}{2}\right)$. Finally, to obtain the coefficients we use the equation obtained in the proof of Theorem 13.

Corollary 16. Consider $n$ a prime integer, $g$ such that $\langle g\rangle=U_{n}$ and the list $b=\left(\beta_{0}, \beta_{1}\right)^{T}$. Then a g-circulant matrix $A$ of order $n$ whose spectrum contains $\left\{\beta_{0}, \pm \beta_{1}\right\}$ is a g-circulant matrix whose first row $\mathbf{c}=\left(c_{0}, c_{1}, \ldots, c_{n-1}\right)^{T}$ has coefficients such that

$$
c_{j}=\frac{1}{n}\left(\beta_{0}+2 \beta_{1} \sum_{\ell=0}^{\frac{n-1}{2}-1} \cos \frac{2(1-j) g^{\ell} \pi}{n}\right)
$$

for $0 \leq j \leq n-1$

Proof. Let $n$ be a prime integer and then $d=n-1=2 m$. Thus $m=\frac{n-1}{2}$ and $k=\frac{n-1}{d}=1$. In consequence, we have only one equivalence class, namely the class of $h_{0}=1$. Finally, by the proof of Theorem 13 the coefficient to be considered has the form as in (16).

The following result gives a necessary and sufficient condition for some lists to become realizable by a $g$-circulant matrix. 
Theorem 17. Let $n$ be a prime integer and $g$ such that $\langle g\rangle=U_{n}$. Then, the list

$$
\Sigma=\left(\beta_{0}, \beta_{1}, \beta_{1} \varphi, \ldots, \beta_{1} \varphi^{n-1}\right)
$$

is realizable by a $g$-circulant matrix $A$ if and only if

$$
\beta_{0} \geq \beta_{1} \geq 0
$$

Proof. For the necessity recall that if $A$ is nonnegative with the components of $\Sigma$ as eigenvalues then $\beta_{0}$ is its spectral radius (see [21]) thus, the inequality in (18) holds. Let $m=\frac{n-1}{2}$. According to the proof of Theorem 13 by using the expressions for the coefficients $c_{j}$, the entries of the first row of the circulant matrix $C=Q_{g}^{-1} A$ are

$$
\begin{aligned}
c_{j} & =\frac{1}{n}\left(\beta_{0}+\beta_{1} \sum_{\ell=0}^{d-1} \tau^{g^{\ell}} \tau^{-g^{\ell} j}\right) \\
& =\frac{1}{n}\left(\beta_{0}+\beta_{1} \sum_{\ell=0}^{n-2}\left(\tau^{1-j}\right)^{g^{\ell}}\right) \\
& =\frac{1}{n}\left(\beta_{0}+\beta_{1} \sum_{\ell=1}^{n-1}\left(\tau^{1-j}\right)^{\ell}\right) \\
& =\frac{1}{n}\left(\beta_{0}+\beta_{1}\left(-1+\frac{1-\left(\tau^{1-j}\right)^{n}}{1-\tau^{1-j}}\right)\right. \\
& =\frac{1}{n}\left(\beta_{0}+\beta_{1}(-1)\right), \quad \text { for } j \neq 1
\end{aligned}
$$

the first part of the result follows. For the second part of the result we need to observe that

$$
n c_{1}=\beta_{0}+(n-1) \beta_{1} .
$$

Therefore, the condition in (18) holds for the matrix $C$ and then $A$ is nonnegative. In consequence, $A$ is a $g$-circulant nonnegative matrix with spectrum as in (17).

Remark 18. If $\beta_{0}=\beta_{1}$ in Theorem 18 the mentioned $g$-circulant nonnegative matrix $A$ becomes $g-\operatorname{circ}\left(0, \frac{\beta_{0}+(n-1) \beta_{1}}{n}, 0, \ldots, 0\right)=g-\operatorname{circ}\left(0, \beta_{0}, 0, \ldots, 0\right)$.

In the following numerical examples we use MATLAB in order to check approximately the orders of the numbers involved in this research because, 
except for Theorem 17, they are displayed in terms of sums of trigonometric functions.

The next example illustrates Corollary 17.

Example 19. Consider the list with 7 elements, $\left(6,5,5 \varphi, 5 \varphi^{2}, 5 \varphi^{3}, 5 \bar{\varphi}^{2}, 5 \bar{\varphi}\right)$, where

$$
\varphi=\cos \frac{2 \pi}{6}+i \sin \frac{2 \pi}{6}=\cos \frac{\pi}{3}+i \sin \frac{\pi}{3} .
$$

In this example it is determined a nonnegative 3-circulant matrix $A$ whose spectrum is the given list. In fact, consider the auxiliary list

$$
\left(6,5 \tau, 5 \tau^{2}, 5 \tau^{3}, 5 \bar{\tau}^{3}, 5 \bar{\tau}^{2}, 5 \bar{\tau}\right)
$$

as the list of eigenvalues of a circulant matrix $C$, with $\tau=\cos \frac{2 \pi}{7}+i \sin \frac{2 \pi}{7}$, and using MATLAB we have

$$
10^{4} C=\operatorname{circ}(1429,51429,1429,1429,1429,1429,1429) .
$$

Let $10^{4} a=1429$, and $10^{4} b=51429$. Again, using MATLAB we obtain the 3-circulant matrix

$$
A=\left(\begin{array}{lllllll}
a & b & a & a & a & a & a \\
a & a & a & a & b & a & a \\
b & a & a & a & a & a & a \\
a & a & a & b & a & a & a \\
a & a & a & a & a & a & b \\
a & a & b & a & a & a & a \\
a & a & a & a & a & b & a
\end{array}\right)
$$

whose eigenvalues are

$$
\Sigma(A)=\{6, \pm 5,2.5 \pm 4.33 i,-2.5 \pm 4.33 i\}
$$

Note that, only the greatest eigenvalue does not have modulus 5 and -5 corresponds to the element $5 \varphi^{3}$ of the given list.

The next example illustrates Corollary 15.

Example 20. Let $n=7, g=6$, we want to find a real 6-circulant matrix $A$ whose spectrum is $\{10, \pm 4, \pm 5, \pm 3\}$. In this case $d=2, k=3$. In order to 
do this the formulae for the coefficients obtained in the proof of the Corollary 15 is used. Thus

$$
\begin{aligned}
c_{j} & =\frac{1}{7}\left(10+8\left(\cos \frac{2(j-1) \pi}{7}+\cos \frac{12(j-1) \pi}{7}\right)+10\left(\cos \frac{4(j-1) \pi}{7}+\cos \frac{24(j-1) \pi}{7}\right)\right) \\
& +6\left(\cos \frac{6(j-1) \pi}{7}+\cos \frac{36(j-1) \pi}{7}\right), \quad 0 \leq j \leq 6 .
\end{aligned}
$$

Using MATLAB we obtain

$$
10^{4} c=(48571,10510,4216,10989,10989,4216,10510) .
$$

\subsection{The order of the subgroup $\langle g\rangle \unlhd U_{n}$ is odd}

Let $g \in U_{n}$ be an integer of odd order and $G$ an additive group. For a subset $S$ of $G$, let $-S$ be defined as

$$
-S=\{-s: s \in S\}
$$

The following result shows that $-1 \notin\langle g\rangle$.

Theorem 21. Let $n>2$ be a prime integer. Let $g$ be an integer such that $(n, g)=1$ and suppose that $|\langle g\rangle|$ is odd. Then $-1 \in h\langle g\rangle$, with $h \neq \equiv 1$.

Proof. If $-1 \in\langle g\rangle$ then the subgroup $H=\{1,-1\} \subseteq\langle g\rangle$. But, by Lagrange theorem, [6], the order of a subgroup of a finite group divides the order of the group and, in this case, 2 should divide the order of $g$ which is a contradiction as $\langle g\rangle$ has odd order.

Let $n>2$ be a prime integer. Then $n-1$ is even, and in consequence, if $g \in U_{n}$ and the subgroup $\langle g\rangle$ has odd order $d$, then the number of equivalence classes $k$ such that (5) holds must be even. Let us suppose that $k=2 m$.

The following result shows that there exists a one to one correspondence among two of the previous equivalence classes.

Theorem 22. Let $n>2$ be a prime integer. Let $g$ be an integer such that $(n, g)=1$ and $|\langle g\rangle|=d$, where $d$ is odd. Then the following holds: $-1 \in h\langle g\rangle$ if and only if $-g^{j} \in h\langle g\rangle, \quad \forall j$ which is equivalent to

$$
-\langle g\rangle=h\langle g\rangle \text { if and only if }-1 \in h\langle g\rangle \text {. }
$$

Proof. Suppose that $-1 \in h\langle g\rangle$. Then $-1=h g^{w}$. Therefore $-g^{j}=$ $\left(h g^{w}\right) g^{j}=h g^{w+j} \in h\langle g\rangle$. Reciprocally, if $-g^{j}=h g^{w}$, for some $w$ then $-1 \sim h$ implying that $-1 \in h\langle g\rangle$.

The next theorem is a consequence of the previous one. 
Theorem 23. Let $n>2$ be a prime integer. Let $g$ be an integer such that $(n, g)=1$ and $|\langle g\rangle|=d$, where $d$ is an odd integer. Let

$$
m=\frac{n-1}{2 d} .
$$

Then, for $h_{t}, h \in U_{n}, \quad-1 \in h\langle g\rangle$ if and only if $-h_{t}\langle g\rangle=h_{t} h\langle g\rangle$. Thus, for $h_{0}=1$,

$$
\begin{aligned}
U_{n} & =\bigcup_{\ell=0}^{m-1}\left(h_{\ell}\langle g\rangle \cup h_{\ell} h\langle g\rangle\right) \\
& =\bigcup_{\ell=0}^{m-1}\left(h_{\ell}\langle g\rangle \cup\left(-h_{\ell}\right)\langle g\rangle\right)
\end{aligned}
$$

is a partition into equivalence classes of the multiplicative group $U_{n}$.

Theorem 24. Let $C$ be a circulant matrix with eigenvalues $\lambda_{0}, \lambda_{1}, \ldots, \lambda_{n-1}$. Under the conditions of Theorem 23, the g-circulant matrix $A=Q_{g} C$ has eigenvalues listed below

$$
\begin{array}{cccccc}
\beta_{0} & \beta_{1} & \beta_{1} \varphi & \beta_{1} \varphi^{2} & \ldots & \beta_{1} \varphi^{d-1} \\
& \bar{\beta}_{1} & \bar{\beta}_{1} \varphi & \bar{\beta}_{1} \varphi^{2} & \ldots & \bar{\beta}_{1} \varphi^{d-1} \\
\vdots & & & & \\
& \beta_{m} & \beta_{m} \varphi & \beta_{m-1} \varphi^{2} & \ldots & \beta_{m} \varphi^{d-1} \\
\bar{\beta}_{m} & \bar{\beta}_{m} \varphi & \bar{\beta}_{m} \varphi^{2} & \ldots & \bar{\beta}_{m} \varphi^{d-1}
\end{array}
$$

where

$$
\beta_{0}=\lambda_{0}
$$

and

$$
\beta_{t+1}=\left(\lambda_{h_{t}} \lambda_{h_{t}} \lambda_{h_{t} g^{2}} \cdots \lambda_{h_{t} g^{d-1}}\right)^{\frac{1}{d}} \in \mathbb{C}, t=0,1, \ldots, m-1,
$$

where $\varphi$ is the primary $d$-th unit root defined in (8),

Now the following inverse eigenvalue problem can be stated.

Problem 25. Given $z_{0} \in \mathbb{R}$ and $m$ arbitrary complex numbers $z_{1}, \ldots, z_{m}$ find a real matrix $A$ g-circulant, for some $g$ whose spectrum contains the complex numbers $z_{0}, z_{1}, \ldots, z_{m}$ and their corresponding complex conjugates. 
To solve Problem 25 we need to establish the following auxiliary problem.

Problem 26. Given $z_{0} \in \mathbb{R}$ and $z_{1}, \ldots, z_{m} \in \mathbb{C}$ find a prime number $n$ such that $n=1+2 m d$, with $d$ odd. Moreover, a positive integer $g$ satisfying identical conditions of those in Theorem 23 and, considering the list

$$
\begin{array}{r}
z_{0}, z_{1}, z_{1} \varphi, z_{1} \varphi^{2}, \ldots z_{1} \varphi^{d-1}, \bar{z}_{1}, \bar{z}_{1} \varphi, \bar{z}_{1} \varphi^{2}, \ldots, \bar{z}_{1} \varphi^{d-1}, \ldots \\
\ldots, z_{m}, z_{m} \varphi, \ldots z_{m} \varphi^{d-1}, \bar{z}_{m}, \bar{z}_{m} \varphi, \ldots \bar{z}_{m} \varphi^{d-1}
\end{array}
$$

find a g-circulant real matrix A whose spectrum is the above list.

As for Problem 10 we need to define an auxiliary list, $\lambda_{0}, \lambda_{1}, \ldots, \lambda_{n}$ displayed in the tables below:

\begin{tabular}{|c|c|c|c|l|c|}
\hline$\lambda_{0}$ & $\lambda_{1}$ & $\lambda_{g}$ & $\lambda_{g^{2}}$ & $\cdots$ & $\lambda_{g^{d-1}}$ \\
\hline & $\lambda_{h_{1}}$ & $\lambda_{h_{1} g}$ & $\lambda_{h_{1} g^{2}}$ & $\cdots$ & $\lambda_{h_{1} g^{d-1}}$ \\
\hline & $\vdots$ & $\vdots$ & $\vdots$ & & $\vdots$ \\
\hline & $\lambda_{h_{m-1}}$ & $\lambda_{h_{m-1} g}$ & $\lambda_{h_{m-1} g^{2}}$ & $\cdots$ & $\lambda_{h_{m-1} g^{d-1}}$ \\
\hline & $\bar{\lambda}_{1}$ & $\bar{\lambda}_{g}$ & $\bar{\lambda}_{g^{2}}$ & $\cdots$ & $\bar{\lambda}_{g^{d-1}}$ \\
\hline & $\bar{\lambda}_{h_{1}}$ & $\bar{\lambda}_{h_{1} g}$ & $\bar{\lambda}_{h_{1} g^{2}}$ & $\cdots$ & $\bar{\lambda}_{h_{1} g^{d-1}}$ \\
\hline & $\vdots$ & $\vdots$ & $\vdots$ & & $\vdots$ \\
\hline & $\bar{\lambda}_{h_{m-1}}$ & $\bar{\lambda}_{h_{m-1} g}$ & $\bar{\lambda}_{h_{m-1} g^{2}}$ & $\cdots$ & $\bar{\lambda}_{h_{m-1} g^{d-1}}$ \\
\hline
\end{tabular}

\begin{tabular}{|c|c|c|c|c|c|}
\hline$z_{0}$ & $z_{1} \tau$ & $z_{1} \tau^{g}$ & $z_{1} \tau^{g^{2}}$ & $\cdots$ & $z_{1} \tau^{g^{d-1}}$ \\
\hline & $z_{2} \tau^{h_{1}}$ & $z_{2} \tau^{h_{1} g}$ & $z_{2} \tau^{h_{1} g^{2}}$ & $\cdots$ & $z_{2} \tau^{h_{1} g^{d-1}}$ \\
\hline & $\vdots$ & $\vdots$ & $\vdots$ & $\vdots$ & $\vdots$ \\
\hline & $z_{m} \tau^{h_{m-1}}$ & $z_{m} \tau^{h_{m-1} g}$ & $z_{m} \tau^{h_{m-1} g^{2}}$ & $\cdots$ & $z_{m} \tau^{h_{m-1} g^{d-1}}$ \\
\hline & $\bar{z}_{1} \tau^{-1}$ & $\bar{z}_{1} \tau^{-g}$ & $\bar{z}_{1} \tau^{-g^{2}}$ & $\cdots$ & $z_{1} \tau^{-g^{d-1}}$ \\
\hline & $\bar{z}_{2} \tau^{-h_{1}}$ & $\bar{z}_{2} \tau^{-h_{1} g}$ & $\bar{z}_{2} \tau^{-h_{1} g^{2}}$ & $\cdots$ & $\bar{z}_{2} \tau^{-h_{1} g^{d-1}}$ \\
\hline & $\vdots$ & $\vdots$ & $\vdots$ & $\vdots$ & $\vdots$ \\
\hline & $\bar{z}_{m} \tau^{-h_{m-1}}$ & $\bar{z}_{m} \tau^{-h_{m-1} g}$ & $\bar{z}_{m} \tau^{-h_{m-1} g^{2}}$ & $\cdots$ & $\bar{z}_{m} \tau^{-h_{m-1} g^{d-1}}$ \\
\hline
\end{tabular}

Theorem 27. Let $n$ and $g$ be positive integers that verify the same conditions as in Theorem 23. Let

$$
\begin{aligned}
& z_{0}, z_{1}, z_{1} \varphi, z_{1} \varphi^{2}, \ldots z_{1} \varphi^{d-1}, \bar{z}_{1}, \bar{z}_{1} \varphi, \bar{z}_{1} \varphi^{2}, \ldots, \bar{z}_{1} \varphi^{d-1}, \ldots, \\
& , \ldots, z_{m}, z_{m} \varphi, \ldots z_{m} \varphi^{d-1}, \bar{z}_{m}, \bar{z}_{m} \varphi, \ldots \bar{z}_{m} \varphi^{d-1}
\end{aligned}
$$


with $z_{0} \in \mathbb{R}$ and $z_{1}, \ldots, z_{m} \in \mathbb{C}$. Then a sufficient condition to find a $g$ circulant real nonnegative matrix $A$ whose spectrum corresponds to the above list, is

$$
\frac{z_{0}}{2} \geq-\operatorname{Re}\left(\mathrm{z}_{1} \sum_{\ell=0}^{\mathrm{d}-1} \tau^{\mathrm{g}^{\ell(1-\mathrm{j})}}+\mathrm{z}_{2} \sum_{\ell=0}^{\mathrm{d}-1} \tau^{\mathrm{h}_{1} \mathrm{~g}^{\ell(1-\mathrm{j})}}+\cdots+\mathrm{z}_{\mathrm{m}} \sum_{\ell=0}^{\mathrm{d}-1} \tau^{\mathrm{h}_{\mathrm{m}-1} \mathrm{~g}^{\ell(1-\mathrm{j})}}\right),
$$

for all $0 \leq j \leq n-1$, where $m=\frac{n-1}{2 d}$ and $\left(h_{1}, h_{2}, \ldots, h_{m-1}\right)$ are defined by (19).

Proof. Consider the auxiliar list of $\lambda^{\prime}$ s defined by (20). Then, by Theorem $\left[9\right.$, Theorem 3.1] the first row $\left(c_{0}, c_{1}, \ldots, c_{n-1}\right)^{T}$ of the circulant matrix of order $n$ whose spectrum is the list in (20) is given by

$$
c_{j}=\frac{1}{n} \sum_{\ell=0}^{n-1} \lambda_{\ell} \tau^{-\ell j}, \quad 0 \leq j \leq n-1 .
$$

Replacing the $\lambda^{\prime}$ s by the list of elements in the statement of the theorem and following the equalities in (20), $c_{j}$ are obtained, for all $0 \leq j \leq n-1$.

$$
\begin{aligned}
c_{j} & =\frac{1}{n}\left(z_{0}+z_{1} \sum_{\ell=0}^{d-1} \tau^{g^{\ell}} \tau^{-g^{\ell} j}+\bar{z}_{1} \sum_{\ell=0}^{d-1} \tau^{-g^{\ell}} \tau^{g^{\ell} j}\right. \\
& +z_{2} \sum_{\ell=0}^{d-1} \tau^{h_{1} g^{\ell}} \tau^{-h_{1} g^{\ell} j}+\bar{z}_{2} \sum_{\ell=0}^{d-1} \tau^{-g^{\ell}} \tau^{g^{\ell} j} \ldots \\
& \left.+z_{m} \sum_{\ell=0}^{d-1} \tau^{h_{k-1} g^{\ell}} \tau^{-h_{k-1} g^{\ell} j}+\bar{z}_{m} \sum_{\ell=0}^{d-1} \tau^{-h_{m-1} g^{\ell}} \tau^{h_{m-1} g^{\ell} j}\right) \\
& =\frac{1}{n}\left(z_{0}+2 \operatorname{Re}\left(\mathrm{z}_{1} \sum_{\ell=0}^{\mathrm{d}-1} \tau^{\mathrm{g}^{\ell}} \tau^{-\mathrm{g}^{\ell_{j}}}+\mathrm{z}_{2} \sum_{\ell=0}^{\mathrm{d}-1} \tau^{\mathrm{h}_{1} \mathrm{~g}^{\ell}} \tau^{-\mathrm{h}_{1} \mathrm{~g}^{\ell_{j}}}+\cdots\right.\right. \\
& \left.+z_{m} \sum_{\ell=0}^{d-1} \tau^{h_{m-1} g^{\ell}} \tau^{-h_{m-1} g^{\ell} j}\right) .
\end{aligned}
$$

Example 28. Let $n=7, g=2, d=3, k=2 m=2$, thus $m=1$. Moreover, consider $z_{0}=10, z_{1}=2-i$. The coefficient vector, $c$, formed by the first 
row of the g-circulant matrix $A$ whose spectrum is the list

$z_{0}, z_{1}, \bar{z}_{1}, z_{1}\left(\frac{-1}{2}+\frac{i \sqrt{3}}{2}\right),-\bar{z}_{1}\left(\frac{1}{2}+\frac{i \sqrt{3}}{2}\right),-z_{1}\left(\frac{1}{2}+\frac{i \sqrt{3}}{2}\right), \bar{z}_{1}\left(\frac{-1}{2}+\frac{i \sqrt{3}}{2}\right)$

has components given by

$$
c_{j}=\frac{1}{7}\left(z_{0}+2 \operatorname{Re}\left(\mathrm{z}_{1}\left(\tau^{(1-\mathrm{j})}+\tau^{2(1-\mathrm{j})}+\tau^{4(1-\mathrm{j})}\right)\right)\right),
$$

for all $0 \leq j \leq 6$. Using MATLAB we obtain that

$$
10^{4} c=(31429,7649,7649,15208,7649,15208,15208) .
$$

Example 29. Let $n=7, g=2, d=3, k=2 m=2$, thus $m=1$. Moreover, consider $z_{0}=2, z_{1}=2$. The coefficient vector, $c$, formed by the first row of the $g$-circulant matrix A whose spectrum is

$$
\{2,2,2,-1+\sqrt{3} i,-1-\sqrt{3} i,-1+\sqrt{3} i,-1-\sqrt{3} i\}
$$

has components given by

$$
c_{j}=\frac{1}{7}\left(2+2 \operatorname{Re}\left(2\left(\tau^{(1-\mathrm{j})}+\tau^{2(1-\mathrm{j})}+\tau^{4(1-\mathrm{j})}\right)\right)\right),
$$

for all $0 \leq j \leq 6$. With the help of MATLAB we obtain that

$$
c=(0,2,0,0,0,0,0) .
$$

\section{Reconstructing certain $g$-circulant matrices from its diagonal entries}

In this section we show that for certain positive integers $n$ and $g$, a $g$-circulant matrix is completely determined by its diagonal entries.

Remark 30. The diagonal vector of the entries of the matrix in (2) is

$$
D(A)=\left(a_{0}, a_{n-g+1}, a_{n-2 g+2}, \ldots, a_{n-(\ell-1) g+\ell-1}, \ldots, a_{g-1}\right)^{T} .
$$

Again, the subscripts are taken $(\bmod \mathrm{n})$. Moreover,

$$
a_{0}=a_{n-0 g+0} \quad \text { and } \quad a_{g-1}=a_{n-(n-1) g+n-1} .
$$


Theorem 31. Let $n$ be a prime integer and let $1<g<n$. Consider

$$
r(A)=\left(a_{0}, a_{1}, \ldots, a_{n-1}\right)^{T}
$$

as the first row vector of a g-circulant matrix A. Then

$$
D(A)=Q_{(n-g+1)} r(A),
$$

where

$$
Q_{(n-g+1)}=(n-g+1)-\operatorname{circ}(1,0, \ldots, 0) .
$$

In consequence,

$$
r(A)=Q_{(n-g+1)^{-1}} D(A) .
$$

Proof. For the subscripts of the diagonal elements of $A$, we note that

$(n-(k-1) g+k-1) \equiv(n-(\ell-1) g+\ell-1)(\bmod n), \quad 0 \leq k<\ell \leq n-1$

if and only if

$$
(\ell-k) \equiv 0(\bmod \mathrm{n}) \text { or } g-1 \equiv 0(\bmod \mathrm{n}) .
$$

The former and latter conditions don't hold because $0<\ell-k \leq n-1<n$ and $g$ is strictly less than $n$, respectively.

Therefore, the diagonal entries of the $g$-circulant matrix $A$ in (2) are a permutation of the first row of $A$. Thus, the difference $(\bmod n)$ between two consecutive subscripts of the vector in (21) is $1-g$, hence (23) and then (24) hold.

Remark 32. From the previous theorem one can say that for a given $n$ prime, $g \in U_{n}$ and, a vector $\mathbf{b}=\left(b_{0}, b_{1}, \ldots, b_{n-1}\right)^{T}$, a g-circulant matrix $A$ can be constructed in such a way that its diagonal elements are the elements of $\mathbf{b}$. Additionally, if in $\mathbf{b}$, one of the coordinates is unknown but the greatest eigenvalue of $A$, say $\beta_{0}$, is known then, a g-circulant matrix $A$ whose diagonal entries are the elements of $\mathbf{b}$ and whose greatest eigenvalue is $\beta_{0}$ can also be constructed. This last statement results from the fact that $\operatorname{tr}(\mathrm{A})=\beta_{0}$ which allows to obtain the unknown coordinate of $\mathbf{b}$.

Example 33. Consider the matrix $A=3-\operatorname{circ}(1,2,3,4,5,6,7)$, whose diagonal is

$$
D(A):=\mathbf{b}=(1,6,4,2,7,5,3)^{T},
$$


moreover

$$
Q_{1+(7-3)}=Q_{5}=\left(\begin{array}{ccccccc}
1 & 0 & 0 & 0 & 0 & 0 & 0 \\
0 & 0 & 0 & 0 & 0 & 1 & 0 \\
0 & 0 & 0 & 1 & 0 & 0 & 0 \\
0 & 1 & 0 & 0 & 0 & 0 & 0 \\
0 & 0 & 0 & 0 & 0 & 0 & 1 \\
0 & 0 & 0 & 0 & 1 & 0 & 0 \\
0 & 0 & 1 & 0 & 0 & 0 & 0
\end{array}\right)
$$

Then

$$
Q_{5} r(A)=(1,6,4,2,7,5,3)^{T}=D(A),
$$

which verifies the equality in (23). We can conclude, in this case, that given the diagonal elements, the first row of the g-circulant matrix (and in consequence all the remaining entries of the g-circulant matrix) can be obtained using the relation in (23) as this relation means that

$$
r(A)=\left(Q_{5}\right)^{-1} D(A) .
$$

Acknowledgments. The authors wish to thank the referee for pointing out the reference [26] where some results in the previous version of this work were already published. We also thank him/her for very useful comments that allow us to improve the paper in another direction.

Enide Andrade was supported by the Portuguese Foundation for Science and Technology (FCT-Fundação para a Ciência e a Tecnologia), through CIDMA - Center for Research and Development in Mathematics and Applications, within project UID/MAT/04106/2019. María Robbiano was partially financed by project VRIDT UCN N 20190403038.

[1] E. Andrade, C. Manzaneda, H. Nina, M. Robbiano, Block matrices and Guo's Index for block circulant matrices with circulant blocks, Lin. Algebra Appl. 556 (2018): 301-322.

[2] E. Andrade, C. Manzaneda, M. Robbiano, Realizable lists on a class of nonnegative matrices, Lin. Algebra Appl. 551 (2018): 36-56.

[3] M. Boyle and D. Handelman, The spectra of nonnegative matrices via symbolic dynamics, Ann. of Math. 133 (1991): 249-316.

[4] P.J. Davis, Circulant matrices: Second Edition, MS Chelsea Publishing Vol. 338, John Wiley and Sons, NY, 1994. 
[5] M. Fiedler, Eigenvalues of nonnegative symmetric matrices, Lin. Algebra Appl. 9 (1974): 119-142.

[6] J. B. Fraileigh, V.J. Katz, A first course in abstract algebra. AddisonWesley 2003.

[7] S. Guo, W. Guo, Perturbing non-real eigenvalues of nonnegative real matrices, Linear Algebra and its Applications 426 (2007): 199-203.

[8] C. Johnson, T. Laffey, R. Loewy, The real and symmetric nonnegative inverse eigenvalue problems are different, Proc. Amer. Math Soc., 12, 124 (1996): 3647-3651.

[9] H. Karner, J. Schneid, C. W. Ueberhuber, Spectral decomposition of real circulant matrices. Lin. Algebra Appl. 367 (2003): 301-311.

[10] T. Laffey, Extreme nonnegative matrices, Lin. Algebra Appl. 275/276 (1998): 349-357. Proceedings of the sixth conference of the international Linear Algebra Society (Chemnitz, 1996).

[11] T. Laffey, Realizing matrices in the nonnegative inverse eigenvalue problem, Matrices and group representations (Coimbra, 1998), Textos Mat. Sér. B, 19, Univ. Coimbra, Coimbra, (1999): 21-31.

[12] T. Laffey, H. Šmigoc, Nonnegative realization of spectra having negative real parts, Linear Algebra Appl., 384 (2004): 199-206.

[13] T. J. Laffey, H. Šmigoc, Nonnegative realization of spectra having negative real parts, Linear Algebra Appl. 416 (2006): 148-159.

[14] T. J. Laffey, H. Šmigoc, Construction of nonnegative symmetric matrices with given spectrum, Linear Algebra Appl. 421 (2007): 97-109.

[15] R. Loewy, D. London. A note on an inverse problem for nonnegative matrices, Lin. and Multilin. Algebra 6, 1 (1978/79): 83-90.

[16] R. Loewy, J. J. Mc Donald, The symmetric nonnegative inverse eigenvalue problem for $5 \times 5$ matrices, Linear Algebra Appl. 393 (2004): 275-298.

[17] R. Loewy, A note on the real nonnegative inverse eigenvalue problem, Electron. J. Linear Algebra, 31 (2016): 765-773. 
[18] C. Manzaneda, E. Andrade, M. Robbiano, Realizable lists via the spectra of structured matrices, Lin. Algebra Appl. 534 (2017): 51-72.

[19] J. Mayo Torre, M. R. Abril, E. Alarcia Estévez, C. Marijuán, M. Pisonero, The nonnegative inverse problem from the coeficientes of the characteristic polynomial EBL digraphs, Linear Algebra Appl. 426 (2007): 729-773.

[20] T. Laffey, E. Meehan, A characterization of trace zero nonnegative $5 \times 5$ matrices, Linear Algebra Appl. 302-303 (1999): 295-302.

[21] H.Minc, Nonnegative Matrices. Wiley, New York, 1988.

[22] P. Paparella, Realizing Suleŭmanova-type spectra via permutative matrices, Electron. J. Linear Algebra, 31 (2016): 306-312.

[23] R. Reams, An inequality for matrices and the inverse eigenvalue problem, Linear and Multilinear Algebra (1996): 367-375.

[24] M. Robbiano, Guo's index for some classes of matrices. Lin. Algebra Appl. 564 (2019): 15-27.

[25] O. Rojo, R. Soto, Existence and construction of nonnegative matrices with complex spectrum. Lin. Algebra Appl. 368 (2003): 53-69.

[26] S. Serra-Capizzano, D. Sesana, A note on the eigenvalues of g-circulants (and of g-Toeplitz, g-Hankel matrices), Calcolo 51 (2014): 639-654.

[27] G. W. Soules, Constructing symmetric nonnegative matrices, Lin. and Multilin. Algebra 13, 3 (1983): 241-251.

[28] H. R. Suleĭmanova, Stochastic matrices with real characteristic numbers, Doklady, Akad. Nuk SSSR (N. S.) 66 (1949): 343-345. 